\title{
GMR
}

\section{miR-125a-5p expression is associated with the age of breast cancer patients}

\author{
H. He ${ }^{1 *}$, F. Xü ${ }^{2 *}$, W. Huang ${ }^{1}$, S.Y. Luo ${ }^{1}$, Y.T. Lin ${ }^{1}$, G.H. Zhang ${ }^{1}$, Q. Du ${ }^{1}$ and \\ R.H. Duan ${ }^{1}$ \\ ${ }^{1}$ The State Key Laboratory of Medical Genetics \& School of Life Sciences, \\ Central South University, Changsha, China \\ 2Department of Breast and Thyroid Surgery, \\ The Second Xiangya Hospital of Central South University, Changsha, China \\ *These authors contributed equally to this study. \\ Corresponding author: R.H. Duan \\ E-mail: duanranhui@sklmg.edu.cn
}

Genet. Mol. Res. 14 (4): 17927-17933 (2015)

Received September 15, 2015

Accepted November 2, 2015

Published December 22, 2015

DOI http://dx.doi.org/10.4238/2015.December.22.17

ABSTRACT. Dysregulated miR-125 observed in multiple cancer types has suggested that it is involved in malignant proliferation and invasion. However, the clinical significance of miR-125 in human breast cancer (BC) has not yet been fully elucidated. In the present study, the expression of miR-125a-5p/3p and miR-125b in 143 pairs of $B C$ and normal adjacent tissues (NATs) was measured by real-time quantitative PCR, and the correlation between expression and clinicopathological features was explored. miR-125a-5p and miR-125b were significantly down-regulated in $\mathrm{BC}$ tissue samples compared with their matched NAT samples, while the difference in miR-125a-3p expression between BC tissues and NATs was not statistically significant. The expression level of miR-125a-5p was found to be significantly higher in younger patients $(<35$ years) than in older patients $(\geq 35, P=0.005)$. When the patients 
were divided into three groups according to age $(<35,36-48$, and $\geq 48$ years), a gradual reduction in miR-125a-5p expression was observed in $B C$ tissue samples that correlated to increases in age $(P=0.009)$. There were no significant correlations between miR-125 expression and other clinicopathological features including tumor size, histological grade, hormone receptor status, Her-2 status, and lymph node metastasis. Taken together, these findings suggest that miR-125a-5p may play an important role in BC progression in an age-dependent manner, and that the down-regulation of miR-125a-5p and miR-125b may serve as independent predictors for breast cancer.

Key words: MicroRNA-125; Breast cancer; Clinicopathology

\section{INTRODUCTION}

Breast cancer $(\mathrm{BC})$ is the most commonly diagnosed cancer in women worldwide, with more than one million new cases diagnosed each year (Turkoz et al., 2013). BC is a heterogeneous disease due to its complicated etiology, which involves both genetic and environmental factors. Although improvements have been madein early detection and treatment for patients, BC is still the leading cause of cancer-related deaths in women (Wang et al., 2013). Therefore, a better understanding of the molecular mechanisms involved in BC development and progression is needed.

Micro-RNAs (miRNAs) are a class of endogenous, noncoding, single-stranded RNA molecules that are approximately 22 nucleotides long (Garzon et al., 2006). They posttranscriptionally regulate gene expression, and as such affect many normal biological processes such as cell proliferation, growth, and survival. Additionally, miRNAs play crucial roles in pathological processes including tumorigenesis, angiogenesis, and tumor invasion (AlvarezGarcia and Miska, 2005). miR-125, which is a highly conserved miRNA found in diverse species from nematodes to humans, and can be found as one of three homologs: hsa-miR-125a, hsamiR-125b-1, and hsa-miR-125b-2 (Shaham et al., 2012). Controversial properties of the members of the miR-125 family have been reported in different types of cancer, such as their potential contribution to the initiation and progression of cancers by acting as either tumor suppressors or oncogenes (Nishida et al., 2011; Bi et al., 2012; Vriens et al., 2012). In BC, miR-125a and miR-125b were reported to be down-regulated in several biopsy specimens, where they acted as tumor suppressors by mediating the ERBB2 and ERBB3 pathways and by targeting the ETS1 gene (Scott et al., 2007). Furthermore, miR-125a represses cell growth by targeting HuR in MCF7 breast cancer cells (Guo et al., 2009), and miR-125b allele-specific regulation of BMPR1B contributes to breast cancer risk (Saetrom et al., 2009). Although functions of the miR-125 family in breast cancer cells have been widely studied in vitro, its involvement in clinical features of BC in vivo has not been fully elucidated.

In the current study, we investigated the expression levels of miR-125a-5p/3p and miR-125b in 143 paired samples of BC and adjacent noncancerous tissues and investigated the potential relationships between miR-125 levels and clinicopathological parameters of BC patients. 


\section{MATERIAL AND METHODS}

\section{Patients and tissues}

Fresh $\mathrm{BC}$ tissues and matched normal adjacent tissues (NATs, $>2 \mathrm{~cm}$ away from the location of the cancer tissue) were collected directly from surgery of consecutive BC patients after removal of the necessary amount of tissue for routine pathology examination at the Second Xiangya Hospital of Central South University (Changsha, China) from December 2009 to March 2011. In total, 143 patients with a median age of 48 years (range 29-77 years) were included in the study. The patient diagnoses included 137 invasive ductal carcinomas, 1 invasive adenosquamous carcinoma, and 5 invasive lobular carcinomas. None of the patients had received chemotherapy or radiotherapy prior to surgery. Tissue samples were immediately frozen in liquid nitrogen after surgical resection and sent directly to the laboratory to perform RNA extraction. The diagnosis and histological grades of each case were independently confirmed by two pathologists based on the World Health Organization tumor classification. Informed written consent was obtained from all patients included in this study, which was approved by the Ethics Committee of the Second Xiangya Hospital of Central South University.

\section{miRNA isolation from tissues}

Total RNA was extracted using TRIzol Reagent (Life Technologies, Carlsbad, CA, USA) according to manufacturer instructions. RNA concentration and purity were determined using a NanoDrop ND-1000 spectrophotometer (NanoDrop Technologies, Wilmington, DE, USA), and samples with a $260 / 280$ ratio of $>1.8$ were considered of acceptable purity. The RNA samples were then stored at $-80^{\circ} \mathrm{C}$ until further use.

\section{qRT-PCR detection of miRNA}

A quantitative real-time PCR (qRT-PCR) assay was performed to detect and quantify mature miR-125. Specifically, SYBR ${ }^{\circledR}$ PrimeScript ${ }^{T M}$ miRNA RT-PCR Kit (TaKaRaBio, Japan) was used to synthesize specific cDNA of miR-125 and RNU6B (as an internal control), and perform qRT-PCR according to the manufacturer protocol. The miRNA-specific forward primers were designed by using the Primer Premier 5.0 software, and the sequences were as follows: miR-125a-5p (5'-TCCCTGAGACCCTTTAACCTGTGA-3'), miR-125a-3p (5'-ACAGGTGAGGTTCTTGGGAGCC-3'), and miR-125b(5'-TCCCTGAGACCCTAACTTGTGA-3'). qRT-PCRs were performed on the CFX96 ${ }^{\mathrm{TM}}$ Real-Time System (Bio-Rad, Hercules, CA, USA). The relative expression of the mature miRNA was normalized to RNU6B and calculated using the comparative cycle threshold $\left(2^{-\Delta \Delta C t}\right)$ method.

\section{Statistical analysis}

Statistical analysis was performed with the SPSS software package, version 17.0 (SPSS Inc., USA). Data on the relative expression of miRNA are reported as means \pm standard deviation. For correlation analysis of BC clinical features with the expression levels of the miR-125 family, two group comparisons were performed using the Mann-Whitney test, and more than two groups 
were compared using one-way analysis of variance (ANOVA). A P value of $<0.05$ was considered as statistically significant. All significance tests were two-sided.

\section{RESULTS}

\section{MiR-125 expression in BC patients}

MiR-125 expression was measured in 143 pairs of tumor tissues and NATs from BC patients, and the expression values obtained were compared between the paired samples. We found that MiR-125a-5p was down-regulated in 67\% (96/143) of BC tissue samples compared with their paired NAT samples. The normalized mean expression \pm SD of miR-125a-5p in BC tumor samples was $0.78 \pm 0.73$ compared with $1.00 \pm 0.64$ in NAT samples (Figure $1 \mathrm{~A}, \mathrm{P}=0.009$ ). miR125b was down-regulated in $90 \%$ (128/143) of BC tissue samples, and the normalized expression in $\mathrm{BC}$ tumor samples was $0.56 \pm 1.36$ compared with $1.00 \pm 1.27$ in NAT samples (Figure $1 \mathrm{~B}, \mathrm{P}<$ 0.001). miR-125a-3p was down-regulated in $55 \%$ (78/143) of $B C$ tissue samples, but the difference in expression between BC tumor samples and NAT samples was not statistically significant $(P=$ 0.656, data not shown).
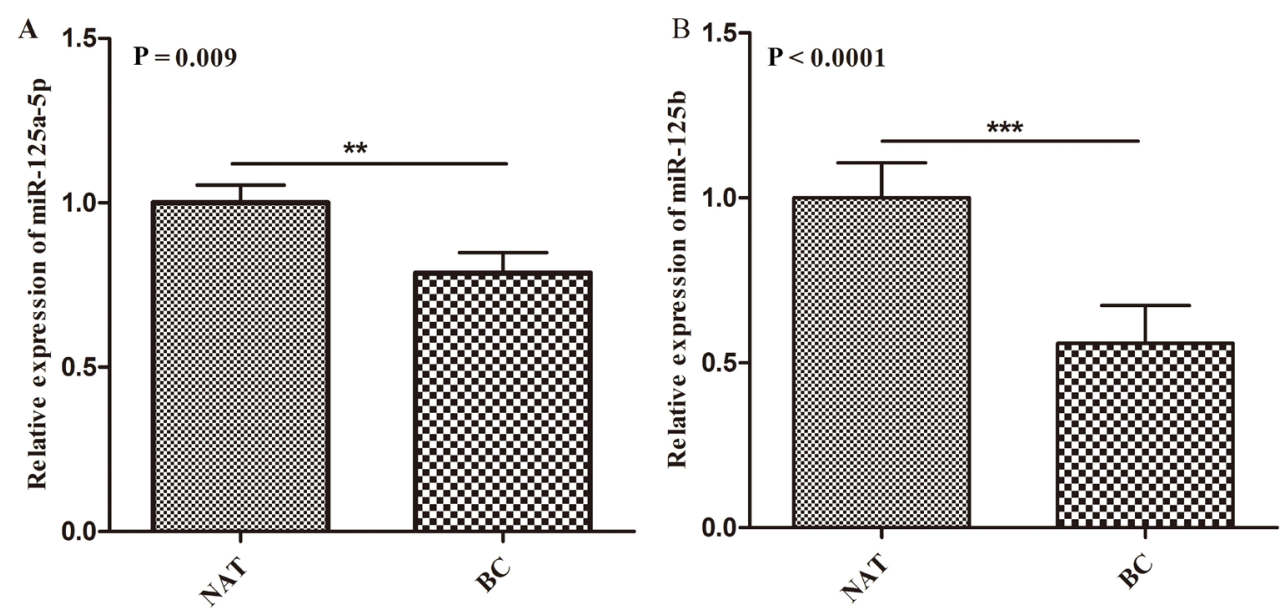

Figure 1. Normalized expression levels of the miR-125 family in breast cancer and normal adjacent tissues. The expression of miR-125a-5p and miR-125b was lower in BC patients than in NATs $(P=0.009$ and $P<0.0001$, respectively). The expression was determined using the $2^{-\Delta \Lambda C t}$ method. BC, breast cancer tissue; NAT, normal adjacent tissue. Bars are reported as mean values with standard deviations.

\section{Correlation of miR-125 expression with clinicopathological features of BC patients}

To investigate the clinical significance of the down-regulated miR-125, the relationships between miR-125 family members and clinicopathological factors were assessed. As shown in Table 1, histological grade, hormone receptor status, Her-2 status, lymph node metastasis, and tumor size were not correlated with miR-125 expression. However, the expression level of miR-125a-5p was significantly higher in younger age patients $(<35)$ than in older patients $(\geq 35, P=0.005)$. To better understand the relationship between miR-125a-5p expression and BC patient age, patients were 
divided into three groups: young BC patients (<35 years, $N=12$ ), young to premenopausal patients (36-48 years, $N=54$ ), and postmenopausal patients ( $\geq 48$ years, $N=77$ ). The results revealed that there was a gradual reduction in the expression of miR-125a-5p with increasing age (Figure 2).

Table 1. Relationship between relative expressions of miR-125 family members and clinicopathological features.

\begin{tabular}{|c|c|c|c|c|c|c|c|}
\hline & \multirow[t]{2}{*}{$\mathrm{N}$} & \multicolumn{2}{|c|}{ miR-125a-5p (2- $\left.{ }^{-\Delta \Delta c t}\right)$ in tumor } & \multicolumn{2}{|c|}{ miR-125a-3p $\left(2^{-\Delta \Delta c t}\right)$ in tumor } & \multicolumn{2}{|c|}{ miR-125b $\left(2^{-\Delta \Delta c t}\right)$ in tumor } \\
\hline & & Mean \pm SD & $P$ value & Mean \pm SD & $P$ value & Mean \pm SD & $P$ value \\
\hline Age (years) & & & 0.005 & & 0.56 & & 0.303 \\
\hline$<35$ years & 12 & $1.62 \pm 0.90$ & & $1.05 \pm 0.35$ & & $0.85 \pm 1.28$ & \\
\hline$\geq 35$ years & 131 & $0.95 \pm 0.75$ & & $1.29 \pm 1.26$ & & $0.50 \pm 0.55$ & \\
\hline Histologic grade & & & 0.911 & & 0.421 & & 0.833 \\
\hline 1 & 9 & $0.89 \pm 0.32$ & & $0.83 \pm 0.57$ & & $0.39 \pm 0.25$ & \\
\hline II & 108 & $1.00 \pm 0.82$ & & $1.29 \pm 1.14$ & & $0.52 \pm 0.65$ & \\
\hline III & 14 & $0.96 \pm 0.62$ & & $1.10 \pm 1.01$ & & $0.53 \pm 0.52$ & \\
\hline Estrogen receptor & & & 0.547 & & 0.399 & & 0.689 \\
\hline Negative & 62 & $0.95 \pm 0.78$ & & $1.45 \pm 1.47$ & & $0.54 \pm 0.54$ & \\
\hline Positive & 78 & $1.03 \pm 0.76$ & & $1.10 \pm 0.90$ & & $0.50 \pm 0.67$ & \\
\hline Progesterone receptor & & & 0.84 & & 0.386 & & 0.638 \\
\hline Negative & 59 & $0.97 \pm 0.81$ & & $1.36 \pm 1.33$ & & $0.49 \pm 0.48$ & \\
\hline Positive & 80 & $1.00 \pm 0.74$ & & $1.19 \pm 1.09$ & & $0.54 \pm 0.70$ & \\
\hline CerbB-2 & & & 0.609 & & 0.911 & & 0.455 \\
\hline Negative & 95 & $0.98 \pm 0.74$ & & $1.27 \pm 1.10$ & & $0.45 \pm 0.40$ & \\
\hline Positive & 18 & $0.89 \pm 0.68$ & & $1.23 \pm 1.78$ & & $0.37 \pm 0.37$ & \\
\hline Lymph node metastasis & & & 0.698 & & 0.681 & & 0.362 \\
\hline Absent & 71 & $0.97 \pm 0.77$ & & $1.23 \pm 1.36$ & & $0.46 \pm 0.47$ & \\
\hline Present & 72 & $1.02 \pm 0.79$ & & $1.31 \pm 1.05$ & & $0.61 \pm 0.76$ & \\
\hline Tumor size & & & 0.743 & & 0.058 & & 0.463 \\
\hline$\leq 2 \mathrm{~cm}$ & 42 & $0.96 \pm 0.70$ & & $1.66 \pm 1.69$ & & $0.58 \pm 0.57$ & \\
\hline$>2 \mathrm{~cm}$ & 97 & $1.00 \pm 0.81$ & & $1.11 \pm 0.92$ & & $0.49 \pm 0.64$ & \\
\hline
\end{tabular}

$\Delta \Delta C T=(C t$ miR-125a-5p - Ct U6) cancer group - (Ct miR-125a-5p - Ct U6) control group.

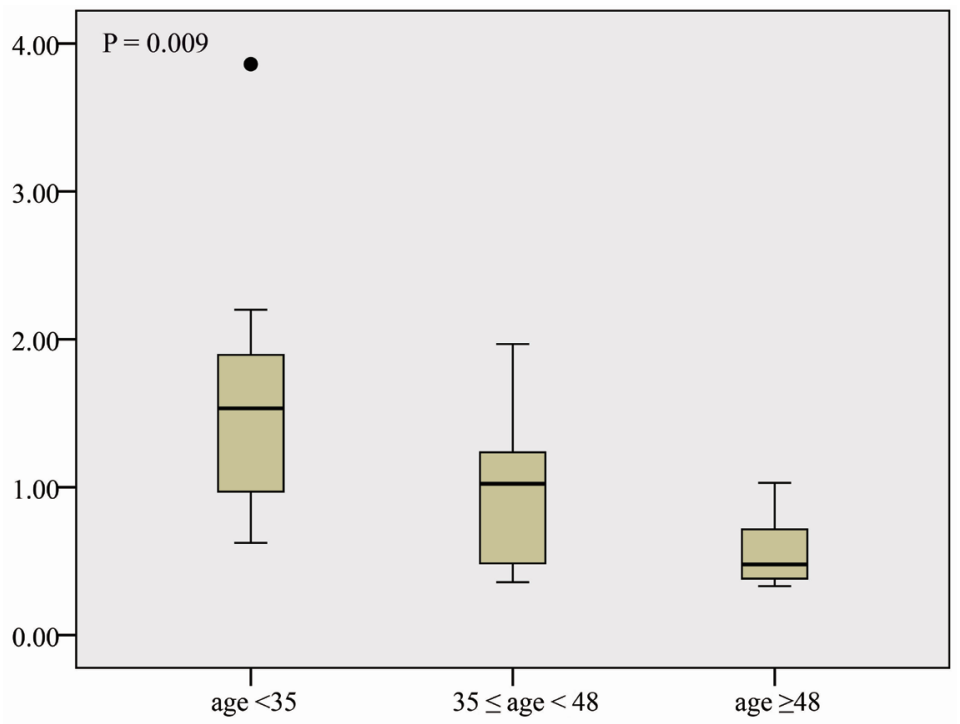

Figure 2. Relative expression of miR-125a-5p for different age groups. Decreased expression of miR-125a-5p is seen with increasing age of breast cancer patients. The relative expression data were analyzed using the $2^{-\Delta \Delta C t}$ method. ANOVA was used to determined significance. 


\section{DISCUSSION}

miR-125 is an important miRNA family, whose members play fundamental roles in development and cell differentiation. Although miR-125 has been found to be down-regulated in $\mathrm{BC}$, its clinical significance had not been investigated in a large patient population. In the present study, we performed a systematic evaluation of the clinical significance of miR-125 in 143 invasive $B C$ cases. We observed a significant down-regulation in miR-125a-5p and miR-125b in BC tissues compared with NATs and further found a gradual reduction in miR-125a-5p expression that corresponded to increased patient age.

miR-125 family members have been demonstrated to be down-regulated in several diseases, highlighting their disease-suppressing properties in such pathologies; however, they also have diseasepromoting properties in certain contexts (Sun et al., 2013). In BC, miR-125b has been demonstrated to be a tumor suppressor by down-regulating ERBB2 and ERBB3. It has further been shown that circulating miR-125b expression is associated with resistance of breast cancer cells, implicating it as a target for anticancer strategies (Wang et al., 2012). Moreover, in contrast to its tumor-suppressive properties, a recent study found that miR-125b could also induce metastasis of human breast cancer cells by targeting STARD13 (StAR-related lipid transfer domain containing 13) (Tang et al., 2012). These controversial properties of the miR-125 in BC suggest that miR-125 plays diverse roles in BC pathogenesis and progression, and therefore the underlying mechanism requires further investigation.

$\mathrm{BC}$ accounts for approximately $23 \%$ of all female malignancies, and while the incidence in younger women is low $(<6 \%)$, these cancers tend to be more aggressive and are associated with unfavorable prognoses (Gabriel and Domchek, 2010; Narod, 2012; Kataoka et al., 2014). Previous studies have compared molecular features of $B C$ to age and have shown that $B C$ tumors in young women have distinct gene expression profiles and deregulated signaling pathways (Anders et al., 2008; Pirone et al., 2012; Colak et al., 2013; Peña-Chilet et al., 2014). The up-regulated miR-125a$5 p$ in younger patients indicates that miR-125a-5p may play an important role in BC development in an age-dependent manner, although its effects and possible targets need to be further elucidated.

In conclusion, our data suggest that miR-125a-5p plays an important role in BC progression according to age and that the down-regulation of miR-125a-5p and miR-125b may serve as independent predictors for breast cancer.

\section{Conflicts of interest}

The authors declare no conflict of interest.

\section{ACKNOWLEDGMENTS}

Research supported in part by the National Natural Science Foundation of China (grants \#81172513 , \#81071028 and \#81571253) and the Program for New Century Excellent Talents (grant \#7603230006)

\section{REFERENCES}

Alvarez-Garcia I and Miska EA (2005). MicroRNA functions in animal development and human disease. Development 132: 4653-4662.

Anders CK, Acharya CR, Hsu DS, Broadwater G, et al. (2008). Age-specific differences in oncogenic pathway deregulation 
seen in human breast tumors. PLoS One 3: e1373.

Bi Q, Tang S, Xia L, Du R, et al. (2012). Ectopic expression of MiR-125a inhibits the proliferation and metastasis of hepatocellular carcinoma by targeting MMP11 and VEGF. PLoS One 7: e40169.

Colak D, Nofal A, Albakheet A, Nirmal M, et al. (2013). Age-specific gene expression signatures for breast tumors and crossspecies conserved potential cancer progression markers in young women. PLoS One 8: e63204.

Gabriel CA and Domchek SM (2010). Breast cancer in young women. Breast Cancer Res. 12: 212.

Garzon R, Fabbri M, Cimmino A, Calin GA, et al. (2006). MicroRNA expression and function in cancer. Trends Mol. Med. 12: 580-587.

Guo X, Wu Y and Hartley RS (2009). MicroRNA-125a represses cell growth by targeting HuR in breast cancer. RNA Biol. 6 : 575-583.

Kataoka A, Tokunaga E, Masuda N, Shien T, et al. (2014). Clinicopathological features of young patients $(<35$ years of age) with breast cancer in a Japanese Breast Cancer Society supported study. Breast Cancer 21: 643-650.

Narod SA (2012). Breast cancer in young women. Nat. Rev. Clin. Oncol. 9: 460-470.

Nishida N, Yokobori T, Mimori K, Sudo T, et al. (2011). MicroRNA miR-125b is a prognostic marker in human colorectal cancer. Int. J. Oncol. 38: 1437-1443.

Peña-Chilet M, Martínez MT, Pérez-Fidalgo JA, Peiró-Chova L, et al. (2014). MicroRNA profile in very young women with breast cancer. BMC Cancer 14: 529.

Pirone JR, D'Arcy M, Stewart DA, Hines WC, et al. (2012). Age-associated gene expression in normal breast tissue mirrors qualitative age-at-incidence patterns for breast cancer. Cancer Epidemiol. Biomarkers Prev. 21: 1735-1744.

Saetrom P, Biesinger J, Li SM, Smith D, et al. (2009). A risk variant in an miR-125b binding site in BMPR1B is associated with breast cancer pathogenesis. Cancer Res. 69: 7459-7465.

Scott GK, Goga A, Bhaumik D, Berger CE, et al. (2007). Coordinate suppression of ERBB2 and ERBB3 by enforced expression of micro-RNA miR-125a or miR-125b. J. Biol. Chem. 282: 1479-1486.

Shaham L, Binder V, Gefen N, Borkhardt A, et al. (2012). MiR-125 in normal and malignant hematopoiesis. Leukemia. 26: 2011-2018.

Sun YM, Lin KY and Chen YQ (2013). Diverse functions of miR-125 family in different cell contexts. J. Hematol. Oncol. $6: 6$.

Tang F, Zhang R, He Y, Zou M, et al. (2012). MicroRNA-125b induces metastasis by targeting STARD13 in MCF-7 and MDAMB-231 breast cancer cells. PLoS One 7: e35435.

Turkoz FP, Solak M, Petekkaya I, Keskin O, et al. (2013). Association between common risk factors and molecular subtypes in breast cancer patients. Breast 22: 344-350.

Vriens MR, Weng J, Suh I, Huynh N, et al. (2012). MicroRNA expression profiling is a potential diagnostic tool for thyroid cancer. Cancer 118: 3426-3432.

Wang H, Tan G, Dong L, Cheng L, et al. (2012). Circulating MiR-125b as a marker predicting chemoresistance in breast cancer. PLoS One 7: e34210.

Wang S, Li H and Wang D (2013).Expression of microRNA-497 and its prognostic significance in human breast cancer. Diagn. Pathol. 21: 172. 